\title{
ON THE COMPLETE INTEGRABILITY OF AN EQUATION HAVING SOLITONS BUT NOT KNOWN TO HAVE A LAX PAIR
}

\author{
A ROYCHOWDHURY and G. MAHATO \\ High Energy Physics Division \\ Jadavpur University \\ Calcutta - 700032, India \\ (Received March 19, 1985)
}

ABSTRACT. It is usually assumed that a system having $\mathrm{N}$-soliton solutions is completely integrable. Here we have analyzed a set of equations occuring in case of capillary gravity waves. Though the system under discussion has N-soliton solutions, it has yet to be shown that the system is completely integrable. No Lax pair is known for the system. Here we show that the system is not completely integrable in the sense of Ablowitz et a1.

KEY WORDS AND PHRASES. Soliton, lax pair, capillar-gravity waves. 1980 AMS SUBJECT CLASSIFICATION CODE. 76.

1. INTRODUCTION.

In recent years there have been tremendous studies for the understanding of the complete integrability of non-linear partial differential equations. Usually equations having $\mathrm{N}$-soliton solutions do possess an Inverse Scattering Transform (IST). But for some equation, it is still not possible to get hold of an IST but one can find $\mathrm{N}$-soliton solution by techniques like those of Hirota. One of the most interesting equations is that of capillary gravity waves initially deduced by KAWAHARA et a1 [1] and analysed for N-soliton solution by Ma [2]. As far as we know no IST has been found for this equation. So here is an example whose solitary wave solutions have been found but whose complete integrability is still unsettled due to the lack of IST. In the current literature there has come out two different [3,4] approaches to test the complete integrability of non-linear partial differential equation. Both of these are really variant of the celebrated Painléve test for the ordinary differential equation. In the approach of Weiss et al, [4] it is required to proceed exactly at every stage of proving the compatibility conditions for the assumed series solution of the nonlinear field variable $\phi(x, t)$. The whole procedure becomes quite tricky and cumbersome after certain stages of calculation. On the other hand in the methodology of Ablowitz et a1 $[5,6]$ it is required to proceed with the leading singularities for the purpose of avoiding moving singularities in the solution manifold; it is only required to determine the position of "resonances" and to obtain the expansion coefficients in arbitrary form. If it can be demonstrated that the expansion coefficients and the wave front of 
the solution manifold is arbitrary then the system is completely integrable. Here we have carried out an analysis of the above mentioned equations (written below in equation (2.1)) from this point of view have concluded that the system is not completely integrable.

2. BASIC EQUATIONS.

The non-linear equations under consideration read

$$
\left.\begin{array}{rl}
i E_{t}+E_{x x} & =n_{E} \\
-i G_{t}+G_{x x} & =n_{G} \\
n_{t}-6 n n_{x} & +n_{x x x}=-(E G)_{x}
\end{array}\right\}
$$

The second of this set is really the complex conjugate of the first one.

Following the procedure of Ablowitz, et al [6] we set

$$
\begin{aligned}
& E=\phi^{b} \sum a_{j} \phi^{j}(x, t) \\
& G=\phi^{q} \sum b_{j} \phi^{j}(x, t) \\
& n=\phi^{s} \sum c_{j} \phi^{j}(x, t)
\end{aligned}
$$

To determine to cominant behavior, we initially assume

$$
\mathrm{E} \sim \phi \mathrm{Pa}_{\mathrm{o}}, \mathrm{G} \sim \phi \mathrm{q}_{\mathrm{b}_{0}}, \quad n \sim \phi^{\mathrm{s}} \mathrm{c}_{\mathrm{o}}
$$

So matching the most singular terms in (2.1) for $\phi(x, t)=0$ we get $s=-2$,

$\mathrm{p}+\mathrm{q}=\mathrm{s}-2=-4$. We proceed with $\mathrm{p}=-2, \mathrm{q}=-2, \mathrm{~s}=-2$. We also get

$$
c_{o}=6, a_{o} b_{o}=-36
$$

Now to determine the next - to - leading order terms, we set,

$$
\begin{aligned}
& E \sim a_{o} \phi^{-2}+a_{r} \phi^{r-2} \\
& G \sim b_{o} \phi^{-2}+b_{r} \phi^{r-2} \\
& n \sim c_{o} \phi^{-2}+c_{r} \phi^{r-2}
\end{aligned}
$$

in the reduced set of equations and obtain

$$
\left.\begin{array}{l}
a_{r}(r-2)(r-3)=a_{r} c_{o}+a_{o} c_{r} \\
b_{r}(r-2)(r-3)=b_{r} c_{o}+b_{o} c_{r} \\
c_{r}(r-2)(r-3)(r-4)=-a_{o} b_{r}(r-4)-a_{r} b_{o}(r-4)
\end{array}\right\}
$$

This set of homogeneous equations can have a non-vanishing solution on $1 y$ if the determinant is zero, that is,

$$
\left|\begin{array}{ccc}
L(r-2)(r-3)-c_{0} & 0 & -a_{0} \\
0 & {\left[(r-2)(r-3)-c_{0}\right]} & -b_{0} \\
b_{0}(r-4) & a_{0}(r-4) & (r-2)(r-3)(r-4)
\end{array}\right|=0
$$

Using equations (2.3), we get the resonance positions at

$$
r=0,-1,4,5,6
$$

As has been elaborately discussed in the paper by Ablowitz et al., the resonance at $r=-1$ corresponds to the arbitrariness of wavefront.

3. DETERMINATION OF COEFFICIENTS AT RESONANCE POSITIONS.

We now proceed to determine the coefficients at the resonance positions. With no loss of generality we assume $\phi(x, t)=x-f(t)$ and all the co-efficients $a_{j}, b_{j}$ and $c_{j}$ are functions of $t$ on $1 y$. We then have

$$
c_{0}=6, a_{0} b_{0}=-36
$$

Let $a_{0}=h(t)$ which is an arbitrary function of $t$. Hence $b_{0}=-\frac{36}{h(t)}$.

For, $-j>1$ we now consider the recurrence relation obtained by linearization with 
respect to the non-leading terms

$$
\begin{aligned}
& \left(\begin{array}{ccc}
(j-2)(j-3)-c_{0} & 0 & -a_{o} \\
0 & (j-2)(j-3)-c_{0} & -b_{o} \\
b_{0}(j-4) & a_{0}(j-4) & (j-2)(j-3)(j-4)
\end{array}\right)\left(\begin{array}{l}
a_{j} \\
b_{j} \\
c_{j}
\end{array}\right) \\
= & \left(\begin{array}{cc}
i(j-3) & a_{j-1} \dot{f}-i \dot{a}_{j-2} \\
i(j-3) & b_{j-1} \dot{f}-i \dot{b}_{j-2} \\
(j-4) & c_{j-2} \dot{f}-\dot{c}_{j-3}
\end{array}\right)
\end{aligned}
$$

For $j=3$ (which is not a resonance position) we have

$$
\left(\begin{array}{ccc}
-6 & 0 & -a_{0} \\
0 & -6 & -b_{0} \\
-b_{0} & -a_{0} & 0
\end{array}\right)\left(\begin{array}{l}
a_{3} \\
b_{3} \\
c_{3}
\end{array}\right)=\left(\begin{array}{c}
-i \dot{a}_{1} \\
i \dot{b}_{1} \\
-c_{1} \dot{f}
\end{array}\right)
$$

which yields

$$
\begin{aligned}
c_{3} & =\frac{1}{72}\left[\frac{9}{5 h}(\dot{h} \dot{f}+h \ddot{f})-\frac{361}{55} i \dot{f}+\frac{361}{55} \frac{f \dot{h}}{h}+\frac{54}{5} i f \dot{f}\right], \\
a_{3} & =\frac{1}{6}\left[i \dot{a}_{1}-h c_{3}\right], \\
b_{3} & =\frac{1}{6}\left[i \dot{b}_{1}-\frac{36}{h} c_{3}\right] .
\end{aligned}
$$

where $c_{3}$ is given by the expression (3.3).

Though $j=3$ is not a resonance these coefficients $a_{3}, b_{3}, c_{3}$ will be needed in our later calculation. Similar calculations were performed for $a_{i}, b_{i}, c_{i} ; i=1,2$.

Though these equations give the coefficients $a_{3}, b_{3}$ and $c_{3}$ explicitly yet the appearance of the arbitrary function $h(t)$ in each of them, introduces some arbitraryness in them.

At the resonance $j=4$, we get the following matrix equation

$$
\left(\begin{array}{ccc}
-4 & 0 & -a_{0} \\
0 & -4 & -b_{0} \\
0 & 0 & 0
\end{array}\right)\left(\begin{array}{c}
a_{4} \\
b_{4} \\
c_{4}
\end{array}\right)=\left(\begin{array}{c}
i a_{3} \dot{f}-i \dot{a}_{2} \\
i b_{3} \dot{f}+i \dot{b}_{2} \\
-\dot{c}_{1}
\end{array}\right)
$$

This gives

$$
\begin{aligned}
& -4 a_{4}-a_{0} c_{4}=i a_{3} \dot{f}-i \dot{a}_{2} \\
& -4 b_{4}-b_{0} c_{4}=i b_{3} \dot{f}+i \dot{b}_{2}
\end{aligned}
$$

and no equation for $c_{4}$, along with

$$
\dot{c}_{1}=0
$$

which has the consequence of fixing the function $f(t)$. So we try to keep nonleading terms in equation (3.4) which is modified to:

$$
\left(\begin{array}{ccc}
-4 & 0 & -a_{0} \\
0 & -4 & -b_{0} \\
0 & 0 & 0
\end{array}\right)\left(\begin{array}{c}
a_{4} \\
b_{4} \\
c_{4}
\end{array}\right)=\left(\begin{array}{c}
i a_{3} \dot{f}-i \dot{a}_{2} \\
i b_{3} \dot{f}-i \dot{b}_{2} \\
-\dot{c}_{1}+12 c_{1} c_{3}
\end{array}\right)
$$

from which we get

$$
\left.\begin{array}{l}
-4 a_{4}-a_{0} c_{4}=i a_{3} \dot{f}-i \dot{a}_{2} \\
-4 b_{4}-b_{0} c_{4}=i b_{3} \dot{f}+i \dot{b}_{2}
\end{array}\right\}
$$

where $c_{4}$ is arbitrary along with $\dot{c}_{1}=12 c_{1} c_{3}$.

The differential equation connecting $h$ and $f$, which originated from the non-trivial solution of $\mathrm{c}_{4}$ is

$$
\dot{\mathrm{f}}=\frac{3}{10}(\dot{\mathrm{h}} \dot{\mathrm{f}}+\mathrm{h} \ddot{\mathrm{f}}) f-\frac{361}{360} \dot{\mathrm{f}} \mathrm{f}+\frac{361}{360} \frac{\mathrm{f} \dot{\mathrm{h}}}{\mathrm{h}}+\frac{9 \dot{\mathrm{i}}}{5} \mathrm{f}^{2} \dot{\mathrm{f}}
$$


At $j=5$, we get the following relation:

$$
\left(\begin{array}{ccc}
0 & 0 & -a_{0} \\
0 & 0 & -b_{0} \\
b_{0} & a_{0} & 6
\end{array}\right) \quad\left(\begin{array}{c}
a_{5} \\
b_{5} \\
c_{5}
\end{array}\right)=\left(\begin{array}{c}
2 i a_{4} \dot{f}-i \dot{a}_{3} \\
2 i b_{4}+i \dot{b}_{3} \\
c_{3}-\dot{c}_{2}
\end{array}\right)
$$

This yields

$$
\begin{aligned}
& -a_{0} c_{5}=2 i a_{4} \dot{f}-i \dot{a}_{3} \\
& -b_{0} c_{5}=2 i b_{4} \dot{f}+i b_{3} \\
& b_{0} a_{5}+a_{0} b_{5}+6 c_{5}=c_{3} \dot{f}-\dot{c}_{2}
\end{aligned}
$$

But equation two values of $c_{5}$ we get an equation

$$
c_{5}=-\frac{1}{a_{0}}\left(2 i a_{4} \dot{f}-i \dot{a}_{3}\right)=-\frac{1}{b_{0}}\left(2 i b_{4} \dot{f}+i \dot{b}_{3}\right)
$$

when substituted from equations (3.7) this leads to another equation for the functions $f(t)$ and $h(t)$, and hence coupled with (3.8) determine $f$ and $h$. So the arbitrariness in all the coefficients and the wave front are lost.

For the resonance at $=6$, we get

$$
\left(\begin{array}{ccc}
6 & 0 & -a_{0} \\
0 & 6 & -b_{0} \\
2 b_{0} & 2 a_{0} & 24
\end{array}\right)\left(\begin{array}{c}
a_{6} \\
b_{6} \\
c_{6}
\end{array}\right)=\left(\begin{array}{c}
3 i a_{5} \dot{f}-i \dot{a}_{4} \\
3 i b_{5} \dot{f}+i \dot{b}_{4} \\
2 c_{4} \dot{f}-\dot{c}_{3}
\end{array}\right)
$$

That is

$$
\begin{gathered}
6 a_{6}-a_{0} c_{6}=3 i a_{5} \dot{f}-i \dot{a}_{4} \\
6 b_{6}-b_{0} c_{6}=3 i b_{5} \dot{f}+i \dot{b}_{4} \\
2 b_{0} a_{6}+2 a_{0} b_{6}+24 c_{6}=2 c_{4} \dot{f}-\dot{c}_{3}
\end{gathered}
$$

Combining these equations we get another differential equation between $h$ and $f$ and this leads to an inconsistency when compaired with the relation (3.8). So that at the resonance positions the compatibility condition is not satisfied.

CONCLUSION: In the above discussions, we have argued that the system described by equation (2.1) is not completely integrable in the sense of Ablowitz et al. [6], and the system is not known to have inverse scattering transform. So one can arise a serious question: If a system has N-soliton solution, does it have a Lax pair always? Our present notion of n.p.d.e's having soliton solution may be very limited and may have to be extended in the future.

\section{REFERENCES}

1. KAWAHARA, T., SUgImOTO, N., and KAKUTANI, T. J. Phys. Soc. Japan 39 (1975).

2. MA, Y. Stud. App. Math. 60 (1979), 73.

3. ABLOWITZ, M.J., RAMAMI, A. and SEgUR, H. Lett. Nuovo Cimento 23 (1980), 333.

4. WEISS, J., TABOR, M. and CARNEVAlE, G. J. Math. Phys. 24(3) (1983), 522.

5. ABLOWITZ, M.J. RAMANI, A. and SEgUR, H. J. Math. Phys. 21 (1980), 715, 1006.

6. ABLOWITZ, M.J. and SEGUR, H. Solitons and the Inverse Scattering Transform, (SIAM, Philadelphia), $19 \overline{81 .}$ 


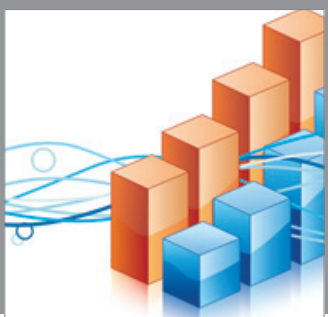

Advances in

Operations Research

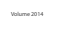

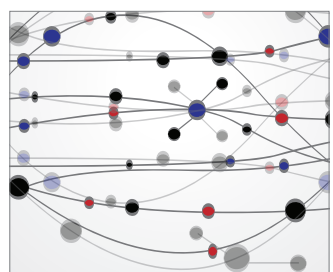

\section{The Scientific} World Journal
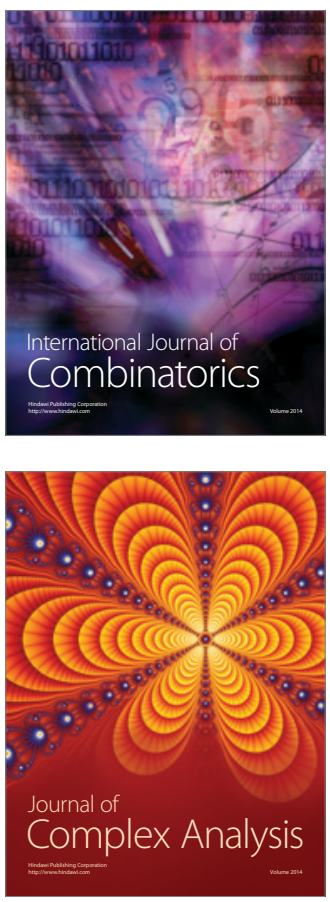

International Journal of

Mathematics and

Mathematical

Sciences
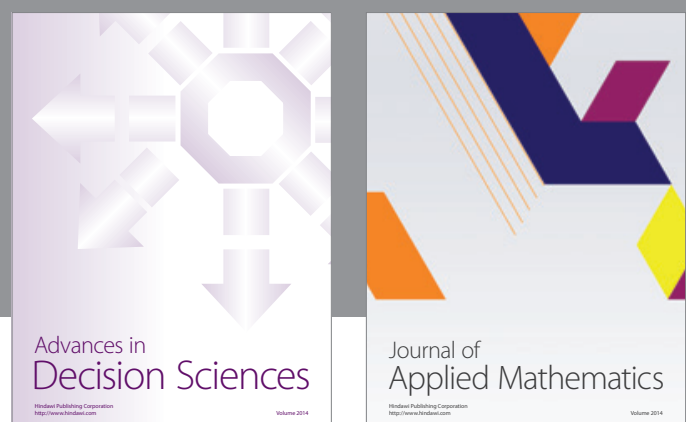

Journal of

Applied Mathematics
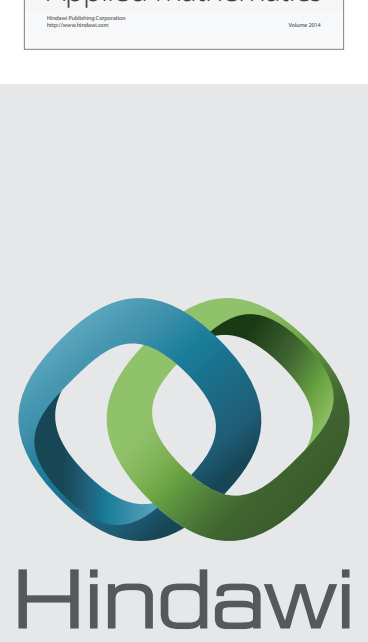

Submit your manuscripts at http://www.hindawi.com
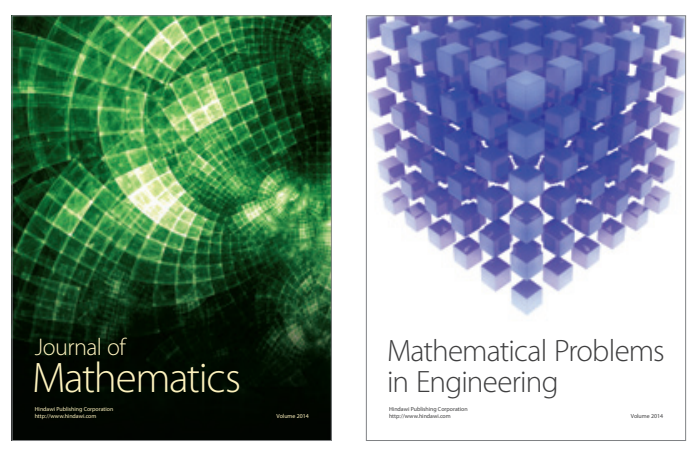

Mathematical Problems in Engineering
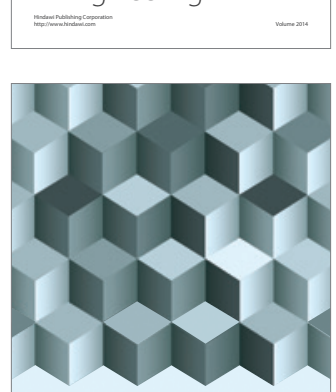

Journal of

Function Spaces
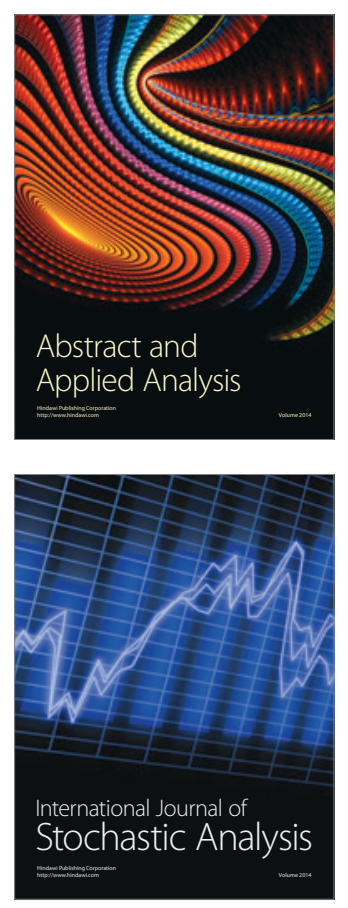

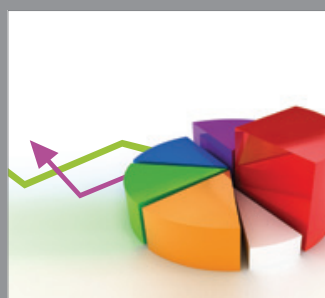

ournal of

Probability and Statistics

Promensencen
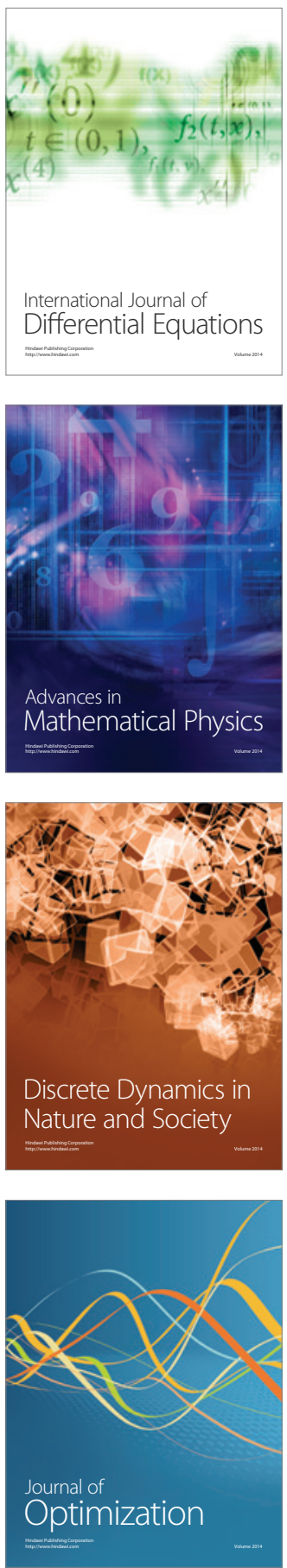